\title{
SEAMLESS MULTIMEDIA TRANSMISSION DURING FAST HANDOVER IN MOBILE IPV6 ENVIRONMENT
}

\author{
Jin Ryong Kim \\ Digital Content Research Div. \\ ETRI \\ Daejeon, KOREA \\ jessekimeetri.re.kr
}

\author{
Youn-Hee Han \\ School of Internet-Media \\ Korea University of Technology \\ and Education, KOREA \\ yhhan@kut.ac.kr
}

\author{
Kwang Hyun Shim \\ Digital Content Research Div. \\ ETRI \\ Daejeon, KOREA \\ shimkheetri.re.kr
}

\begin{abstract}
In this paper, we propose the seamless video transmission scheme over wireless LANs during fast handover in Mobile IPv6. In fact, a mobile node cannot receive the IP packets during handover. A number of extensions to fast handover for mobile IPv6 have been introduced to recover its weakness. Fast handover algorithm in Mobile IPv6 allows the new access router in the adjacent cell to pre-buffer the data packets of a mobile node to prevent packet loss. In a mobile communication environment, however, transmission of multimedia data should consider the realtimeliness of the data. We focus on the multimedia data delivery during fast handover in Mobile IPv6 environment.
\end{abstract}

\section{INTRODUCTION}

Recent technologies in Mobile IPv6 (MIPv6) have made it possible to provide IP addresses to mobile devices [1]. MIPv6 [2, 3] is designed to manage the movements of mobile nodes among wireless IPv6 networks. In Mobile IP, a mobile device always has a Home Agent and has to be provided the new care-of-address (NCoA) whenever it moves from one network to another network. However, one interesting issue is the delay imposed upon mobile nodes when switching between limited wireless cells. In other words, the mobility of a mobile node generates the latency during the handover between wireless cells and it causes IP packet loss or packet delay. To reduce the handover latency, a large amount of effort has been expended over the several years. In this paper, we focus on one of the proposals called Fast Handover for MIPv6 (FMIPv6) [4].

FMIPv6 allows the new access router in the adjacent cell to pre-buffer the data packets of a mobile node to prevent packet loss. When the mobile node is about to enter the cell in new access router while it communicates with the corresponding node, the new access router receives and keeps the data packets from the corresponding node via the previous access router. Once the mobile node enters the cell in new access router, the new access router retransmits the buffered data packet to the mobile node so that the packet loss can be minimized during the handover.

In a mobile communication environment, however, realtime transmission of multimedia data is not guaranteed [6]. Thus, the transmission of streaming media must consider the realtimeliness of the data. The packet loss, delay, or jitter would disturb the realtimeliness of media data and they can bring out a number of problems when retrieving the media data.

In this paper, we propose a framework to seamlessly transmit realtime media data during fast handover under RTP protocol [7-9]. The streaming media packets over RTP from corresponding node (i.e. media server) will be transmitted and cached under the new access router via tunneling during the mobile node is handed-over. Once the mobile node gets the new cell, the new access router starts releasing cached media data. In section 2 we summarize MIPv6 and its extension FMIPv6. Then, in the following section, we introduce our video transmission scheme over RTP in FMIPv6 environment. In the next section, we carry out the simulation-based test to demonstrate the effectiveness of our scheme. Finally, we give some concluding remarks in the last section.

\section{SYNOPSIS: FAST HANDOVER IN MOBILE IPV6}

In this section, we explain MIPv6 and how the mobile node performs the fast handover.

Figure 1 illustrates handover in MIPv6. MIPv6 manages mobile nodes movements between wireless network subnets. Each mobile node (MN) is identified by its home address although it may be connecting to through another network. When connecting through a foreign network, a MN sends its location information to a home agent, which intercepts packets intended for the device and tunnels them to the current location. However, it cannot use its home address anymore to send packets in the new subnet. Thus, it needs to acquire NCoA in the new subnet. 
Fast handover in MIPv6 (FMIPv6) [4] describes a protocol to replace MIPv6 movement detection algorithm and $\mathrm{NCoA}$ configuration procedure. It enables $\mathrm{MN}$ to quickly detect that it is now moving to a new subnet by providing the new access point (NAP) identifier and receiving the associated subnet prefix information. $\mathrm{MN}$ also formulates a prospective $\mathrm{NCoA}$, if at all possible, when still present on current subnet. Furthermore, in order to make the $\mathrm{MN}$ allocate the $\mathrm{NCoA}$ to its interface immediately after attaching to the new subnet, FMIPv6 provides the NCoA confirmation procedure, which is executed before or during MN switches its subnet.

The scenario in which an MN receives the positive result about the confirmation of its prospective NCoA on the current subnet is called predictive mode. The scenario in which an $\mathrm{MN}$ checks the uniqueness of $\mathrm{NCoA}$ after $\mathrm{MN}$ attaches to new subnet is called reactive mode. Although $\mathrm{MN}$ initiates the NCoA confirmation at early time on the current subnet, FMIPv6 would fall into reactive mode if $\mathrm{MN}$ could not receive the confirmation result on the current subnet. In addition, if the proposed $\mathrm{NCoA}$ is rejected during the NCoA confirmation procedure, $\mathrm{MN}$ may configure $\mathrm{NCoA}$ by itself after movement so that handover latency becomes long. In order to achieve more reduction of handover latency, it is required that predictive mode should occur more frequently than reactive mode. So, it is very demanded that the NCoA confirmation should be done promptly and its result should be always successful.

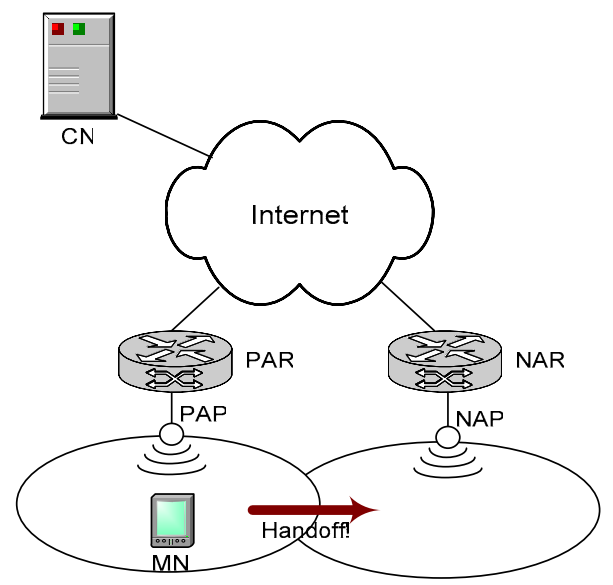

Fig. 1. Handover in mobile IPv6 environment

In FMIPv6, fast handover initiation is based on an indication from a wireless Layer 2 trigger (or Layer 2 signal), which informs that $\mathrm{MN}$ will soon be handed over. To start a fast-handover, MN sends a Router Solicitation for Proxy (RtSolPr) message to the previous access router (PAR). The RtSolPr contains the Layer 2 identifier of a target AP which $\mathrm{MN}$ will move to. At this time, PAR maps the Layer 2 identifier into proper target NAR (New Access Router). MN will receive in response a Proxy Router
Advertisement (PrRtAdv) message from PAR with NAR's network prefix. Based on the response, $M N$ forms a prospective NCoA, and immediately sends Handover Initiation (HI) message to NAR, which initiates the process of establishing a bidirectional tunnel between PAR and NAR.

This HI contains the prospective NCoA. NAR starts its NCoA confirmation procedure as soon as it receives HI. If the period of confirmation procedure would be long, (hereby, the delivery of Handover Acknowledgement (Hack) and Fast Binding Acknowledgement (FBAck) would be delayed), MN might not receive FBAck before it disconnects with PAR. This means FMIPv6 falls into the reactive mode, and $\mathrm{MN}$ should (re)send FBU as soon as it attaches to NAR. As a result, at this case, it requires additional FBU delivery, which will be encapsulated in Fast Neighbor Advertisement (FNA), with the consumption of wireless bandwidth.

\section{VIDEO TRANSMISSION SCHEME IN FAST HANDOVER FOR MOBILE IPV6}

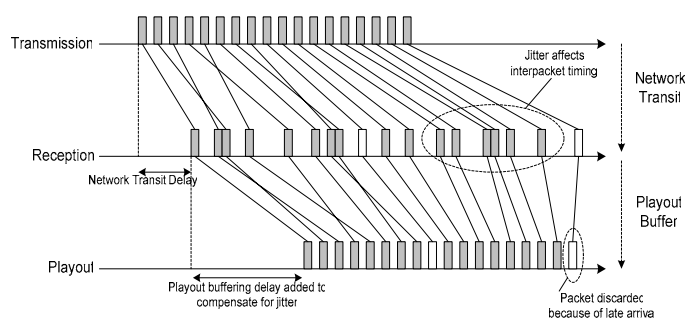

Fig. 2. Network jitter in RTP

In this work, we seamlessly transmit realtime multimedia data on FMIPv6. New access router (NAR) pre-buffers streaming packets from corresponding node (i.e. media server) during $\mathrm{MN}$ is handed-over. That is, NAR receives media and its control packets (RTP packets) from corresponding node via tunnel during the handover, and effectively caches and bypasses them to $\mathrm{MN}$ after the handover.

Figure 2 illustrates network jitter in RTP. In general, one of the problems in RTP streaming is the jitter of the packet and buffer overflow and underflow in mobile devices. It is because the RTP data packets travel along the different network paths so that the sequence of packet order can be altered and the inter-packet timing cannot be guaranteed. As a result, the media contents will be distorted or delayed when the mobile device retrieves the media data. Further, it will cause buffer overflows in playout buffer of the mobile node if the new access router bypasses all the cached data once it starts to communicate with the mobile node without considering characteristics of RTP under such circumstances. Also the mobile node should take care of processing overhead because it has to reorganize RTP sequence. 
We reorganize the RTP packets buffered in NAR according to RTP sequence. Further, we consider the sequence order and RTP timestamp and release RTP packets to the mobile node by resizing sliding window depending on packet size. The piled up packets are reorganized and readjusted their intervals. At this time, NAR stores information of RTP packets and transmits buffered packets using sender's report (SR) to $\mathrm{MN}$.

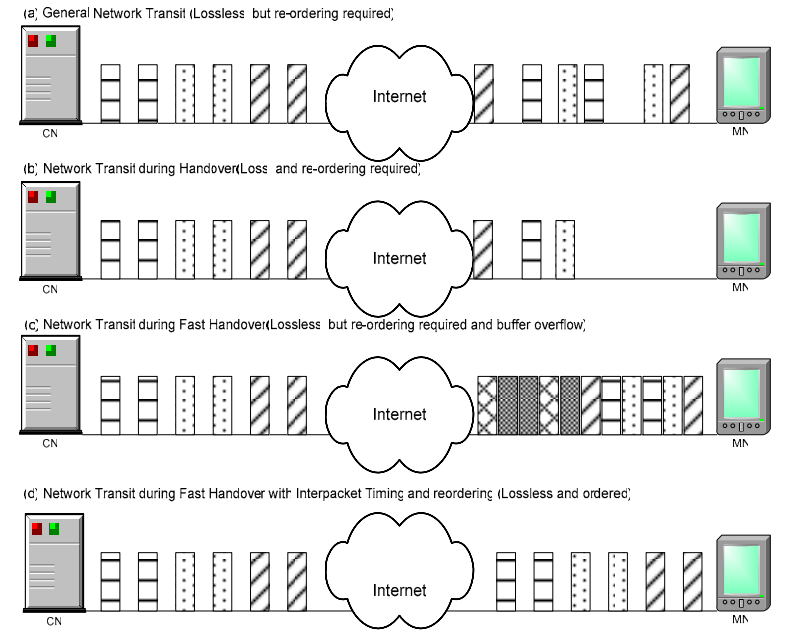

Fig. 3. Handover in mobile IPv6 environment

Figure 3(a) illustrates the general network transit of packets. The packets are transmitted from $\mathrm{CN}$ with fixed interval. Once they traverse the Internet, the arrival time for each packet is changed and consequently jitter occurs. Figure 3(b) illustrates network transit during handover. When MN moves from one cell to another, the packets can be lost or delayed during moving time and this will seriously influence the media playback. However, this can be solved by using FMIPv6 algorithm. That is, the packets can be tunneled and buffered in NAR during handover and these buffered packets can be retransmitted to $\mathrm{MN}$ when it receives FNA as illustrated in Figure 3(c). However, all buffered packets are transmitted at the same time and this will cause packet overflow and packet delay in MN's playout buffer as illustrated in Figure 4. This is because NAR does not consider any packet intervals, it will violate the realtimeliness of media and consequently other packets can be also damaged.

We improve the mechanism of transmission to achieve the seamless transmission of media packets through the following conditions.

Condition 1. MN receives media data using RTP packets from $\mathrm{CN}$ in Foreign Network, and it retrieves them in realtime.

Condition 2. When MN moves from Previous Network to Foreign Network, MN communicates with PAR and moves into the cell of NAR.

Condition 3. PAR copies recent SR (Sender's Report) of RTCP.
Condition 4. We assume that the period of RTCP is 5 seconds.

Condition 5. RTP packets are tunneled, transmitted and buffered in NAR.

Condition 6. NAR buffers RTP packets tunneled between NAR and PAR in RTP sequence order. Moreover, NAR stores the arrival time, sequence number, RTP timestamp, SSRC of each packet into its database. It also stores video and audio separately.

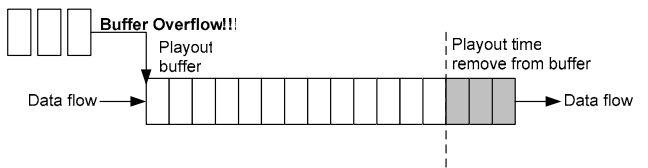

Fig. 4. Buffer overflow in playout buffer of MN

Figure 5 illustrates how we effectively deliver tunneled RTP packets in FMIPv6. When MN moves from the cell of PAR to the cell of NAR and at the same time it receives RTP packets from CN, PAR stores a recent copy of SR in periodical RTCP messages. When the indication of Layer 2 trigger (or Layer 2 signal) occurs, PAR sends SR with HI to NAR. After initiating all the messages (e.g. HI, Hack, RA, FBU), the tunnel between PAR and NAR is established. Once the tunnel is set up, NAR stores RTP packets into its audio and video buffers and stores information including SR message, arrival time of RTP packets, sequence numbers, timestamps, and SSRC into its database. When the packets are stored in the buffer of NAR, they are stored in order of sequence number. However, the timestamps for some packets can be identical as illustrated in Figure 6. Whenever NAR receives FNA from $M N$, NAR adjust time interval using timestamp and NTP time of SR from its database. Rearranged RTP packets then are sent to MN. PAP (Previous Access Point) and NAP (New Access Point) are the access points for their routers.

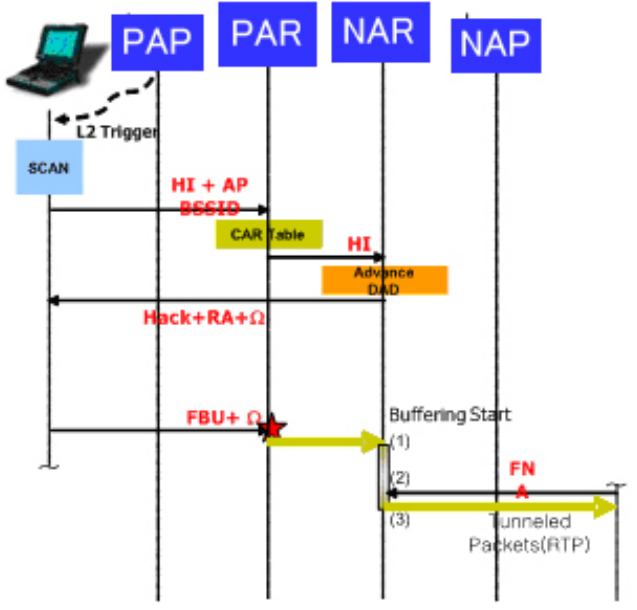

Fig. 5. Procedure of fast handover and RTP delivery 


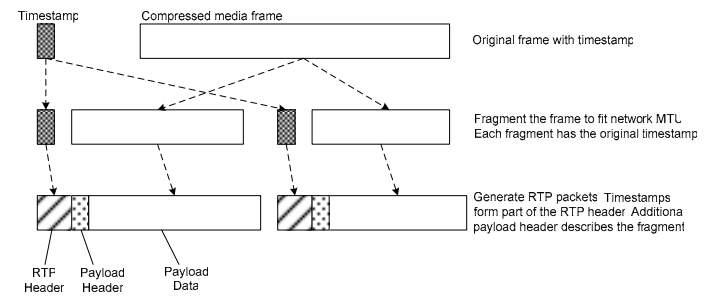

Fig. 6. Fragmentation of media frame into RTP packets

\section{PERFORMANCE EVALUATION}

In this section, we present a simulation result through the implementation of seamless multimedia transmission scheme over FMIPv6 that we proposed. Simulation is conducted using Darwin Streaming Server version 5.5.1[10] as $\mathrm{CN}$; and media file has H.264 encoded with $1 \mathrm{Mbit} \mathrm{Hz}$. The size of media is $380 \times 480$ pixels with $48,000 \mathrm{kHz}$ of audio. We set 5 seconds of handover time.

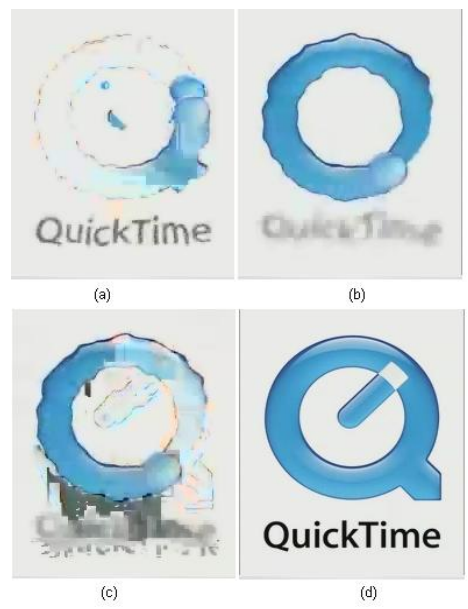

Fig. 7. Simulation results: (a) and (b) playback time $: 2 \mathrm{sec}$, (c) and (d) playback time : $49 \mathrm{sec}$

Figure 7 illustrates the result of simulation. Figure 7(a) and (c) are the screen shots when MN receives all the RTP packets after it sends FNA to NAR. This is because NAR does not consider any arrangement of RTP packets nor time intervals, the buffer of $\mathrm{MN}$ is overflowed and jitter is occurred. In Figure 7(b) and (d), we apply our transmission scheme on NAR. It is noticed that media has clearly displayed without any fluctuation.

\section{ACKNOLEDGEMENTS}

We would like to acknowledge the contributions of numerous colleagues at ETRI and Samsung Advanced Institute of Technology who have contributed to our work and thinking in this area. In particular, we would like to thank Hee Jin Jang, Bum Hyun Lim, Il Kyu Park, Jae Yong Chung, and Jung Youl Lim.

\section{CONCLUSION}

In this work, we were able to eliminate jitters, packet loss, and packet delay effectively when MN moves to the cell of NAR. Because NAR has shorter hop to MN, it is more advantageous than $\mathrm{CN}$ adaptively adjusting packets. Furthermore, because NAR buffers tunneled packets in order, MN does not have to conduct packet loss detection or rearrange them, and consequently additional loads on $\mathrm{MN}$ can be reduced. As a result, it is found that $\mathrm{MN}$ can retrieve realtime media seamlessly during handover in FMIPv6 by reordering RTP packets.

\section{REFERENCES}

[1] James D. Solomon, Mobile IP: The Internet Unplugged, Prentice-Hall, Inc., Upper Saddle River, New Jersey, USA, 19961997.

[2] D. Johnson, C. Perkins, and J. Arkko, "Mobility Support in IPv6," Internet Engineering Task Force (IETF) draft-ietf-mobileipipv6-24.txt (work in progress), June 2003.

[3] S.-J. Vaughan-Nichols, "Mobile IPv6 and The Future of Wireless Internet Access," IEEE Computer, Vol. 36, Issue 2, pp. 18-20, February 2003.

[4] Youn-Hee Han, Seung-Hee Hwang, and HeeJin Jang, "Design and Evaluation of an Address Configuration and Confirmation Scheme for IPv6 Mobility Support," Proc. of IEEE Wireless Communications and Networking Conference, IEEE, Altalnta, Georgia, USA, pp. 1270-1275, 21-25 March 2004.

[5] Nicholas Montavont and Thomas Noel, "Analysis and Evaluation of Mobile IPv6 Handovers over Wireless LAN", Mobile Networking and Applications (MONET), special issue on Mobile Networking through IPv6 or IPv4, Volume 8, Number 6, November 2003.

[6] Abbas Jamalipour, The Wireless Mobile Internet: Architectures, Protocols and Services, John Wiley \& Sons Ltd, The Atrium, Southern Gate, Chichester, West Sussex, England, 2003.

[7] H. Schulzrinne. "RTP Profile for Audio and Video Conferences with Minimal Control," Internet Engineering Task Force, RFC 1890, January 1996.

[8] Colin Perkins, RTP: Audio and Video for the Internet, Pearson Education, Inc., 75 Arlington Street, Suite 300, Boston, MA, USA, June 2003

[9] T. Koren, S. Casner, J. Geevarghese, B. Thompson, and P. Ruddy, "Enhanced Compressed RTP (CRTP) for Links with High Delay, Packet Loss and Reordering," Internet Engineering Task Force, Work in Progress (Update to RFC 2508), February 2003.

[10] Apple, http://developer.apple.com/darwin/ 